\title{
Genetic polymorphisms of short tandem repeat loci D13S305, D13S631 and D13S634 in the Han population of Tianjin, China
}

\author{
YUNFANG SHI, XIAOZHOU LI, DUAN JU, YAN LI, XIULING ZHANG and YING ZHANG \\ Laboratory of Medical Genetics, Department of Obstetrics and Gynecology, \\ Tianjin Medical University General Hospital, Tianjin 300052, P.R. China \\ Received June 18, 2014; Accepted January 26, 2015
}

DOI: $10.3892 /$ etm.2015.2560

\begin{abstract}
Short tandem repeat (STR) markers, also known as microsatellites, are extensively used in mapping studies, forensics and disease diagnosis due to their small dimension and low mutation and high polymorphism rates. In recent years quantitative fluorescence polymerase chain reaction (QF-PCR) has been successfully used to amplify STR markers in the prenatal diagnosis of common chromosomal abnormalities. This method provides a diagnosis of common aneuploidies 24-48 h after sampling with low error rates and cost; however, the size of different alleles, frequency, heterozygosity and distribution of STR markers vary among different populations. In the present study three STR markers, D13S305, D13S631 and D13S634, on chromosome 13 were analyzed in 350 unrelated individuals (200 males and 150 females) from the Han population of Tianjin, China using QF-PCR. Eleven, seven and 11 alleles of each marker were observed, respectively. The frequencies of the genotypes were in good agreement with Hardy-Weinberg equilibrium $(\mathrm{P}>0.05)$. The results showed that these three STR markers were highly polymorphic in the Han population of Tianjin, China. The study has provided basic data for use in the prenatal diagnosis of Patau syndrome.
\end{abstract}

\section{Introduction}

Patau syndrome, also known as trisomy 13 , is the third most frequently occurring autosomal trisomy among newborns, with a prevalence of 1 in 4,000 live births and 1

Correspondence to: Professor Ying Zhang, Laboratory of Medical Genetics, Department of Obstetrics and Gynecology, Tianjin Medical University General Hospital, 154 Anshan Road, Tianjin 300052, P.R. China

E-mail: yingzhangdoc@126.com

Key words: Patau syndrome, short tandem repeat, quantitative fluorescence polymerase chain reaction in 29,000 total births (1). The condition was first described by Smith et al (2) in 1960. The conventional method utilized to establish a prenatal diagnosis of Patau syndrome involves cytogenetic analysis through amniocentesis or chorionic villous sampling; however, this is time-consuming (14-21 days), labor-intensive, requires skilled analysis and is not suitable for the large-scale screening of all pregnant females $(3,4)$.

Short tandem repeat (STR) loci, also known as microsatellites or simple sequence repeats, are extensively used in mapping studies, forensics and disease diagnosis due to their small dimension and low mutation and high polymorphism rates (5). The use of quantitative fluorescence polymerase chain reaction (QF-PCR) for the amplification of STR markers is, to date, the most developed method for the rapid and high sensitivity and specificity screening of chromosomal aneuploidies involving chromosomes 21, 18, 13, X and $Y(3,4,6,7)$. Trisomies are identified by detecting three doses of an STR. The clinical utility of the QF-PCR assay in several prenatal centers of Europe has been confirmed by its high sensitivity and specificity within $24-48$ h $(3,4,6,7)$. One of the most evident advantages of QF-PCR is the automation of the procedure, allowing a high throughput of samples at an extremely low cost; however, the allele frequency and heterozygosity of STR markers vary among different populations. It is important to select STR markers and analyze their genetic polymorphisms prior to using them in prenatal diagnosis in order to minimize the percentage of uninformative QF-PCR results and increase the diagnostic accuracy (8-10).

Although several studies have reported the application of QF-PCR in the prenatal diagnosis of common chromosomal aneuploidies, the majority of the markers used were based on a Caucasian population and were not suitable for a Chinese population (3,4,6-10). Furthermore, few studies have investigated the polymorphisms of STR markers on chromosome $13(4,7,10)$. The present investigation was carried out to study the genetic polymorphisms of three STR markers, D13S305, D13S631 and D13S634, on chromosome 13 in the Han population of Tianjin, China. To the best of our knowledge, no data for these STR markers are currently available for this population. 


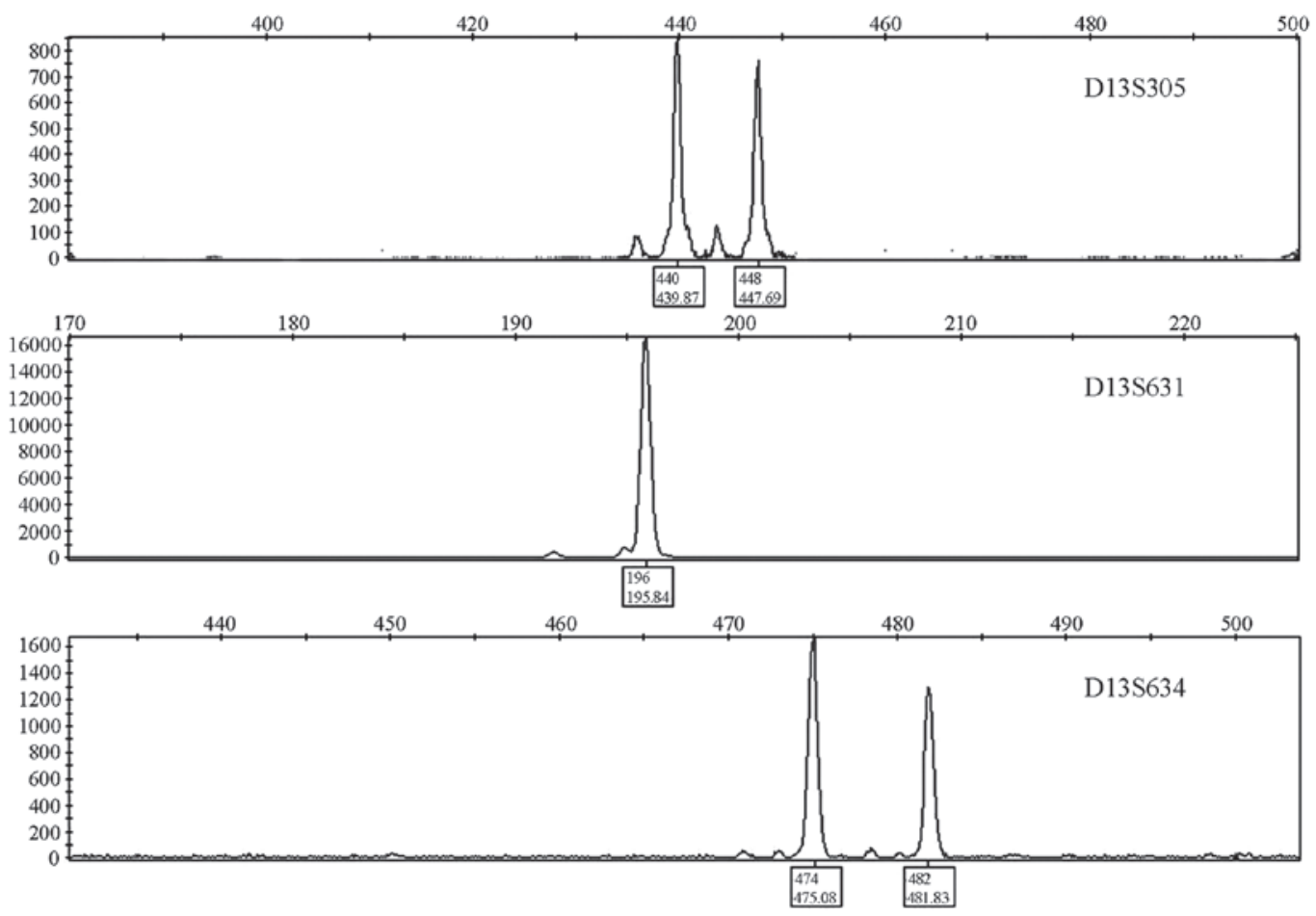

Figure 1. A sample showing a heterozygous pattern for D13S305 and D13S634 and a homozygous pattern for D13S631.

\section{Materials and methods}

Samples. Samples from 350 unrelated individual (200 males and 150 females) from the Han population of Tianjin, China were collected by the Laboratory of Medical Genetics of the Tianjin Medical University General Hospital (Tianjin, China) between September 2010 and August 2012. The parents and grandparents of the subjects were also from the same population. Routine karyotyping with standard G-binding was performed on all samples to exclude chromosomal abnormalities and the results showed that the karyotype of all samples was normal.

This study was conducted in accordance with the Declaration of Helsinki and with approval from the Ethics Committee of Tianjin Medical University General Hospital. Written informed consent was obtained from all participants.

DNA extraction and PCR amplification. Genomic DNA was extracted from the peripheral blood samples using a Whole Blood Genomic DNA Isolation kit (Beijing Dingguo Changsheng Biotechnology Co., Ltd., Beijing, China) according to the manufacturer's instructions. The characteristics of the STR markers D13S305, D13S631 and D13S634 are listed in Table I (http://www.ncbi.nlm.nih.gov/unists). Each STR marker was amplified individually. PCR was carried out in a total reaction volume of $25 \mu \mathrm{l}$, containing $100 \mathrm{ng}$ genomic DNA, $0.1 \mu \mathrm{mol} / 1$ each primer, $1 \mathrm{X}$ PCR buffer (containing $\mathrm{MgCl}_{2}$ ), $0.8 \mu \mathrm{mol} / 1$ deoxynucleotide triphosphate and 1 unit Taq polymerase (Beijing Dingguo Changsheng Biotechnology Co., Ltd.). The amplification was performed in a GeneAmp ${ }^{\circledR}$ PCR System 9600 (Perkin Elmer, Waltham, MA, USA). The protocol for the PCR was as follows: Initial denaturation at $95^{\circ} \mathrm{C}$ for 5 min and 35 cycles consisting of denaturation at $95^{\circ} \mathrm{C}$ for $30 \mathrm{sec}$, annealing at $55^{\circ} \mathrm{C}\left(\mathrm{D} 13 \mathrm{~S} 305\right.$ and D13S634) or $56^{\circ} \mathrm{C}$ (D13S631) for $30 \mathrm{sec}$ and extension at $72^{\circ} \mathrm{C}$ for $30 \mathrm{sec}$. The final extension step was $72^{\circ} \mathrm{C}$ for $10 \mathrm{~min}$.

Genotyping. A total of $1.5 \mu \mathrm{l}$ PCR products, $8.5 \mu \mathrm{l}$ deionized formamide and a GeneScan ${ }^{\mathrm{TM}}-500 \mathrm{ROX}$ size standard (Applied Biosystems, Foster City, CA, USA) were mixed and denatured at $94^{\circ} \mathrm{C}$ for $2 \mathrm{~min}$ for capillary electrophoresis using an ABI 3730 sequencer (Applied Biosystems). The results were analyzed using ABI Prism ${ }^{\circledR}$ GeneMapper version 3.0 software (Applied Biosystems), and the relative peak areas were automatically calculated by the software. Two to three alleles of each locus was sequenced for confirmation of repeat number using the ABI 3730 sequencer (Applied Biosystems). The allele nomenclature followed the recommendations of the International Society for Forensic Hemogenetics (11).

Statistical analysis. Allelic frequencies were calculated by direct counting for males and females collectively. Each of the STR markers was checked using the $\chi^{2}$ test to verify the Hardy-Weinberg equilibrium (HWE). Genetic polymorphism data, including expected heterozygosity, observed heterozygosity, polymorphic information content (PIC), power of discrimination (PD), power of exclusion (PE) and matching probability, were calculated using the PowerStat V12 software (Promega Corp., Madison, WI, USA).

\section{Results}

Sample amplification and repeat sequences. All samples were successfully amplified. Heterozygotes showed two peaks with a ratio of 1:1 while homozygotes showed only a single peak (Fig. 1). The D13S305, D13S631 and D13S634 
Table I. Characteristics of the short tandem repeat markers D13S305, D13S631 and D13S634.

\begin{tabular}{lclr}
\hline Locus & Accession number & \multicolumn{1}{c}{ Primer sequence (5'-3') } & Chromosome location \\
\hline D13S305 & L31091 & f-FAM-TTGAGGACCTGTCGTTACG & $13 q 13.3$ \\
& r-TTATAGAGCAGTTAAGGCACA & $13 q 32.1$ \\
D13S631 & f-FAM-GGCAACAAGAGCAAAACTCT & $13 q 14.3-13 q 22$ \\
D13S634 & r-TAGCCCTCACCATGATTGG & f-FAM-TCCAGATAGGCAGATTCAAT & 1363 \\
& & r-CCTTCTTCTTCCCATTGATA & \\
\hline
\end{tabular}

Table II. Repeat sequences of the short tandem repeat markers D13S305, D13S631 and D13S634.

\begin{tabular}{lc}
\hline Locus & Repeat sequence \\
\hline D13S305 & CTTT \\
D13S631 & ATCT \\
D13S634 & AAGA \\
\hline
\end{tabular}

Table III. Allele nomenclature, allele frequencies and the fragment size of the D13S305, D13S631 and D13S634 short tandem repeat markers.

\begin{tabular}{llll}
\hline \multicolumn{3}{c}{ Locus } \\
\cline { 2 - 4 } Allele & D13S305(bp) & D13S631 (bp) & D13S634 (bp) \\
\hline 5 & & & $0.0029(454)$ \\
6 & & $0.0043(458)$ \\
7 & & $0.0043(188)$ & $0.0200(470)$ \\
8 & & $0.0586(192)$ & $0.2171(474)$ \\
9 & & $0.3471(196)$ & $0.0657(478)$ \\
10 & $0.0029(416)$ & $0.2857(200)$ & $0.2900(482)$ \\
11 & $0.0071(420)$ & $0.1386(204)$ & $0.2329(486)$ \\
12 & $0.0100(424)$ & $0.1471(208)$ & $0.1143(490)$ \\
13 & $0.0543(428)$ & $0.0457(494)$ \\
14 & $0.1071(432)$ & & $0.0057(498)$ \\
15 & $0.1929(436)$ & $0.0186(212)$ & \\
16 & $0.2429(440)$ & & \\
17 & $0.1914(444)$ & & \\
18 & $0.1357(448)$ & & \\
19 & $0.0457(452)$ & & \\
20 & $0.0100(456)$ & & \\
\hline & & & \\
\hline
\end{tabular}

Data are presented as frequency (fragment size in bp).

STR markers were all tetranucleotide repeats, and the allele nomenclature was based on the number of repeats. The repeat sequences are shown in Table II. Since no significant differences were found in the allele frequencies between the males and females in the study, the data were computed collectively.
Table IV. Genetic polymorphism data of the D13S305, D13S631 and D13S634 short tandem repeat markers.

\begin{tabular}{lccc}
\hline & \multicolumn{3}{c}{ Locus } \\
\cline { 2 - 4 } Statistic & D13S305 & D13S631 & D13S634 \\
\hline He & 0.833 & 0.754 & 0.796 \\
Ho & 0.809 & 0.714 & 0.757 \\
PIC & 0.810 & 0.714 & 0.765 \\
PD & 0.948 & 0.893 & 0.927 \\
PE & 0.615 & 0.451 & 0.522 \\
MP & 0.052 & 0.107 & 0.073 \\
HWE (P-value) & 0.878 & 0.647 & 0.397 \\
\hline
\end{tabular}

He, expected heterozygosity; Ho, observed heterozygosity; PIC, polymorphic information content; $\mathrm{PD}$, power of discrimination; PE, power of exclusion; MP, matching probability; HWE, Hardy-Weinberg equilibrium.

STR alleles. Eleven, seven and 11 alleles of the D13S305, D13S631 and D13S634 markers were observed, respectively. In total, 29 alleles were observed. Allele 8 was not found among the D13S634 markers analyzed. D13S305 was the most polymorphic marker with 11 different alleles, referred to as alleles 10-20, and higher genetic polymorphism data compared to D13S634. D13S631 was the least polymorphic marker with only seven alleles (referred to as alleles 9-15). Allele nomenclature, frequencies and fragment size of the D13S305, D13S631 and D13S634 STR markers are shown in Table III. No significant deviations from the HWE were observed in these STR markers ( $\mathrm{P}>0.05)$ (Table IV).

Genetic polymorphism data. Genetic polymorphism data for the D13S305, D13S631 and D13S634 STR markers are shown in Table IV. Heterozygosity for the three markers ranged between 0.714 and 0.809 in this study. The results showed that D13S305 was the most informative marker, with heterozygosity at 0.809 , while D13S631 was the least informative marker, with heterozygosity at 0.714 . The PIC values ranged between 0.714 (D13S631) and 0.810 (D13S305), the PD values ranged between 0.893 (D13S631) and 0.948 (D13S305) and the PE ranged between 0.451 (D13S631) and 0.615 (D13S305). The study showed that D13S305 was the most polymorphic marker among these STR markers. 


\section{Discussion}

The use of QF-PCR to amplify STR markers in order to diagnose chromosomal aneuploidies was first applied in 1993 (12). As this approach has developed, it has been widely used in numerous countries for $>10$ years and has proved to be an accurate, less labor-intensive and robust prenatal test for chromosomal aneuploidies (3,4,6-10). One of the most evident advantages of QF-PCR is the automation of the procedure, allowing a high throughput of samples at a low cost. In general, the use of three or four highly polymorphic STR markers per chromosome allows a high diagnostic accuracy in the majority of samples (13). Although the incidence of Patau syndrome is less than that of trisomy 21 (Down syndrome) and trisomy 18 (Edwards syndrome), there have been a number of patients with trisomy 13 who have survived past the first decade, even reaching 146 months (14). Furthermore, few papers have reported the application of the QF-PCR method in detecting trisomy 13 or investigated the polymorphisms of STR markers on chromosome 13. The aim of the present study was to select STR markers on chromosome 13 that were highly polymorphic for the Chinese population and to optimize the PCR system in order to design a novel, multiplex PCR assay to rapidly diagnose chromosomal abnormalities.

Different laboratories applying QF-PCR for prenatal diagnosis typically use individual markers or commercial rapid aneuploidy detection kits (15); however, the majority of these markers have been based on the Caucasian population and are not suitable for the Chinese population, such as D13S742 and D13S258. In our preliminary experiment it was found that the marker D13S742 was not polymorphic in the Han population of Tianjin, China, and that the heterozygosity was only 0.475 . Furthermore, the marker D13S258 failed to amplify. In addition to the level of heterozygosity, the characteristics of the repeats were taken into consideration in the selection of the STR markers. Tetranucleotide repeats were preferred due to the occurrence of shutters and the mutation rate being low (16). The location of the STR markers on the target chromosome is also crucial. In the present study, three STR markers distributed along the long arm of chromosome 13 were selected; these exhibited no overlap, which could have increased the possibility of detecting partial monosomy or trisomy (17). These STR markers, D13S305, D13S631 and D13S634, were selected from a total of eight STR markers, D13S631, D13S634, D13S258, D13S303, D13S256, D13S628, D13S742 and D13S305, based on their extensive use in the literature $(4,7,10,15,17-19)$, data on their repeat number, heterozygosity and genetic polymorphisms and our preliminary experiment, and were shown to be highly polymorphic in the Han population of Tianjin, China.

Previous studies have also analyzed the polymorphism of these three STR markers and used them in prenatal diagnosis. Cirigliano et al (4) found nine, eight and 12 alleles of the D13S305, D13S631 and D13S634 markers in a Spanish and Italian population. The heterozygosity was $0.75,0.78$ and 0.85 , respectively. The authors then performed QF-PCR on 43,000 clinical samples and found 127 cases of trisomy 13, for which the QF-PCR results were consistent with those of the cytogenetic analysis (4). Putzova et al (17) found 11 and eight alleles of D13S305 and D13S631, respectively, in a Czech population, and the heterozygosity of each marker was 0.809 and 0.827. With regard to D13S305 and D13S634, Cho et al (18) found 11 and 10 alleles, respectively, in a Korean population, while Nasiri et al (10) observed nine and eight alleles, respectively, in an Iranian population. The heterozygosity values in the study by Cho et al (18) were 0.84 and 0.74 , respectively, while those in the study by Nasiri et al (10) were 0.7850 and 0.8807 , respectively. Onay et al (15) observed seven, eight and 12 alleles of D13S305, D13S631 and D13S634 markers, respectively, in a Turkish population and found that the heterozygosity was $0.75,0.78$ and 0.85 . One case of trisomy 13 was detected using QF-PCR. Baig et al (19) found nine and 11 alleles of D13S631 and D13S634 in a Singaporean population with heterozygosity at 0.768 and 0.839 , respectively. In the same study, QF-PCR was performed to detect aneuploidies; 47 autosomal trisomies and 16 gender chromosome aneuploidies were identified, including 30 cases of trisomy 13.

In conclusion, the present study has shown that the D13S305, D13S631 and D13S634 STR markers are highly polymorphic in the Han population of Tianjin, China and has provided basic data that can be used in human population genetic studies. These STR markers can be applied in the prenatal diagnosis of Patau syndrome.

\section{References}

1. Lin HY, Lin SP, Chen YJ, et al: Clinical characteristics and survival of trisomy 13 in a medical center in Taiwan, 1985-2004. Pediatr Int 49: 380-386, 2007.

2. Smith DW, Patau K, Therman E and Inhorn SL: A new autosomal trisomy syndrome: multiple congenital anomalies caused by an extra chromosome. J Pediatr 57: 338-345, 1960.

3. Faas BH, Cirigliano V and Bui TH: Rapid methods for targeted prenatal diagnosis of common chromosome aneuploidies. Semin Fetal Neonatal Med 16: 81-87, 2011.

4. Cirigliano V, Voglino G, Ordoñez E, et al: Rapid prenatal diagnosis of common chromosome aneuploidies by QF-PCR, results of 9 years of clinical experience. Prenat Diagn 29: 40-49, 2009.

5. Tariq MA, Ullah O, Riazuddin SA and Riazuddin S: Allele frequency distribution of $13 \mathrm{X}$-chromosomal STR loci in Pakistani population. Int J Legal Med 122: 525-528, 2008.

6. Hills A, Donaghue C, Waters J, et al: QF-PCR as a stand-alone test for prenatal samples: the first 2 years' experience in the London region. Prenat Diagn 30: 509-517, 2010.

7. Papoulidis I, Siomou E, Sotiriadis A, et al: Dual testing with QF-PCR and karyotype analysis for prenatal diagnosis of chromosomal abnormalities. Evaluation of 13,500 cases with consideration of using QF-PCR as a stand-alone test according to referral indications. Prenat Diagn 32: 680-685, 2012.

8. Quaife R, Wong LF, Tan SY, Chua WY, Lim SS, Hammersley CJ and Yeo HL: QF-PCR-based prenatal detection of aneuploidy in a southeast Asian population. Prenat Diagn 24: 407-413, 2004.

9. Andonova S, Vazharova R, Dimitrova V, Mazneikova V, Toncheva D and Kremensky I: Introduction of the QF-PCR analysis for the purposes of prenatal diagnosis in Bulgaria - estimation of applicability of 6 STR markers on chromosomes 21 and 18. Prenat Diagn 24: 202-208, 2004.

10. Nasiri H, Noori-Dalooi MR, Dastan J and Ghaffari SR: Investigation of QF-PCR application for rapid prenatal diagnosis of chromosomal aneuploidies in Iranian population. Iran J Pediatr 21: 15-20, 2011.

11. Bär W, Brinkmann B, Budowle B, et al: DNA recommendations. Further report of the DNA Commission of the ISFH regarding the use of short tandem repeat systems. International Society for Forensic Haemogenetics. Int J Legal Med 110: 175-176, 1997.

12. Mansfield ES: Diagnosis of Down syndrome and other aneuploidies using quantitative polymerase chain reaction and small tandem repeat polymorphisms. Hum Mol Genet 2: 43-50, 1993.

13. Jain S, Panigrahi I, Sheth J and Agarwal S: STR markers for detecting heterogeneity in Indian population. Mol Biol Rep 39: 461-465, 2012. 
14. Iliopoulos D, Sekerli E, Vassiliou G, Sidiropoulou V, Topalidis A, Dimopoulou D and Voyiatzis N: Patau syndrome with a long survival (146 months): a clinical report and review of literature. Am J Med Genet A 140: 92-93, 2006.

15. Onay H, Ugurlu T, Aykut A, et al: Rapid prenatal diagnosis of common aneuploidies in amniotic fluid using quantitative fluorescent polymerase chain reaction. Gynecol Obstet Invest 66: 104-110, 2008.

16. Nadeem A, Babar ME, Hussain M and Tahir MA: Development of pentaplex PCR and genetic analysis of X chromosomal STRs in Punjabi population of Pakistan. Mol Biol Rep 36: 1671-1675, 2009.
17. Putzova M, Pecnova L, Dvorakova L, Soldatova I, Goetz P and Stejskal D: OmniPlex - a new QF-PCR assay for prenatal diagnosis of common aneuploidies based on evaluation of the heterozygosity of short tandem repeat loci in the Czech population. Prenat Diagn 28: 1214-1220, 2008.

18. Cho EH, Park BY, Kang YS and Lee EH: Validation of QF-PCR in a Korean population. Prenat Diagn 29: 213-216, 2009.

19. Baig S, Ho SS, Ng BL, et al: Development of quantitative-fluorescence polymerase chain reaction for the rapid prenatal diagnosis of common chromosomal aneuploidies in 1,000 samples in Singapore. Singapore Med J 51: 343-348, 2010. 